\title{
DEVELOPMENT OF A FUSION APPROACH SELECTION TOOL
}

\author{
C. Pohl ${ }^{\mathrm{a}, *}$, Y. Zeng ${ }^{\mathrm{b}}$ \\ ${ }^{a}$ Geoscience and Digital Earth Centre (GeoDEC), Universiti Teknologi Malaysia (UTM), Blok T06/FGHT, 81310 Johor Bahru, \\ Malaysia - c.pohl@utm.my \\ ${ }^{\mathrm{b}}$ Chinese Academy of Surveying and Mapping, 28 Lianhuachixi Road, Haidian District, Beijing 100830, P.R.China - \\ zengyu@casm.ac.cn
}

Commission II, WG VII/6

KEY WORDS: image fusion, remote sensing, research, database, tool

\begin{abstract}
:
During the last decades number and quality of available remote sensing satellite sensors for Earth observation has grown significantly. The amount of available multi-sensor images along with their increased spatial and spectral resolution provides new challenges to Earth scientists. With a Fusion Approach Selection Tool (FAST) the remote sensing community would obtain access to an optimized and improved image processing technology. Remote sensing image fusion is a mean to produce images containing information that is not inherent in the single image alone. In the meantime the user has access to sophisticated commercialized image fusion techniques plus the option to tune the parameters of each individual technique to match the anticipated application. This leaves the operator with an uncountable number of options to combine remote sensing images, not talking about the selection of the appropriate images, resolution and bands. Image fusion can be a machine and time-consuming endeavour. In addition it requires knowledge about remote sensing, image fusion, digital image processing and the application. FAST shall provide the user with a quick overview of processing flows to choose from to reach the target. FAST will ask for available images, application parameters and desired information to process this input to come out with a workflow to quickly obtain the best results. It will optimize data and image fusion techniques. It provides an overview on the possible results from which the user can choose the best. FAST will enable even inexperienced users to use advanced processing methods to maximize the benefit of multi-sensor image exploitation.
\end{abstract}

\section{INTRODUCTION}

Due to the increased number of earth observation programmes in different countries many new satellites have been launched in the past decades. With their launch terabytes of new data and images have become available to the user community. Most missions carry more than one sensor on board enabling the exploitation of multi-sensor data to increase information content and quality. Some missions, such as COSMO SkyMed SAR, follow multiplatform concepts reducing the re-visit time and mission capabilities tremendously (Battiston et al. 2014). All in all, users face large data choices for their areas of interest and applications.

Remote sensing image fusion has become a recognized tool for multi-sensor information extraction. Image Fusion is the combination of a group of images with the objective of producing a single image of greater quality and reliability (Li et al. 1993). The past 20 years of research in this field has resulted in established operational algorithms yielding high quality results. Most popular is the so-called 'pansharpening', where lower spatial resolution multispectral images are fused with a high-resolution panchromatic channel. Its popularity results from the fact that most optical sensors carry abilities to acquire multispectral and panchromatic data simultaneously so that the problems of changes in time, platform and orbital differences causing errors in the fused image cease to exist. It aims at preserving multispectral quality while increasing the spatial resolution without introducing artefacts (Xu et al. 2015). However, the benefit of complementary sensor data, such as visible and infrared (VIR) combined with synthetic aperture radar (SAR) has shown its potential, too. Fused VIR/SAR images lead to more reliable information extraction using classification apart from the fact that it eases SAR image interpretation (Werner et al. 2014; Otukei et al. 2015). The development and improvement of image fusion algorithms are strong research fields and form an on-going process. Currently five groups of techniques are applicable to earth observation images (Pohl and van Genderen 2015):

1. Component substitution,

2. Numerical and statistical image fusion,

3. Modulation-based techniques,

4. Multi-resolution approaches, and

5. Hybrid techniques.

Component substitutions (CS) use a transformation, converting the original data into a new space. The fusion takes place in the process of replacing one of the resulting components with the new image to be combined. A reverse transform returns the final fused image. An example is the traditional intensity - hue - saturation (IHS) transform and its derivatives. The second group comprises all mathematical combinations with Brovey Transform (BT) as its most popular representative. The principal component analysis (PCA) also belongs to this group. The third group contains high pass filtering (HPF) and smoothing filter-based intensity modulation (SFIM). Both apply filters to modulate lower resolution multispectral images. A very strong cluster with constantly increasing applications is the set of multi-resolution approaches (MRA). Their popularity has come with the increased power of computers and software. Wavelet transform (WT), contourlet transform (CT) and other

* Corresponding author 
decomposition techniques belong to this group. Last but not least more and more research is going into hybrid algorithms where the benefits of two or more fusion algorithms are combined into one process. Examples are the Ehlers method (IHS and Fast Fourier Transform) (Ehlers 2004), IHS-BT (Zhang and Hong 2005) or IHS-WT (Hong et al. 2009).

Different data and different algorithms require different preprocessing. Optical remote sensing images undergo atmospheric correction while SAR images should be de-speckled to remove the inherent noise. Both data have to be co-registered and geometrically corrected to coincide on a pixel-by-pixel basis. In the correction and fusion process resampling is necessary, which requires a choice of resampling methods. Problems in coregistration and the type of resampling method can have a negative impact on the fusion results (Baronti et al. 2011; Jawak and Luis 2013).

\section{A FUSION APPROACH SELECTION TOOL}

Multi-sensor image fusion is a machine and time-consuming endeavour. The use of image fusion in remote sensing image exploitation leads to many opportunities based on the variability in input and targeted outcome (Pohl and van Genderen 2015). The choice of an appropriate fusion technique depends on many factors. It largely alters with the type and quality of accessible data, the image processing software and user capabilities, the storage capacity, the application and desired information, just to name a few. It requires knowledge about the sensors, the algorithm, and the application for which the information is provided. Combining the available number of satellites and sensors with the options to fuse the images we obtain an amount of possibilities that cannot be handled by a single user anymore. The problem is illustrated in figure 1 .

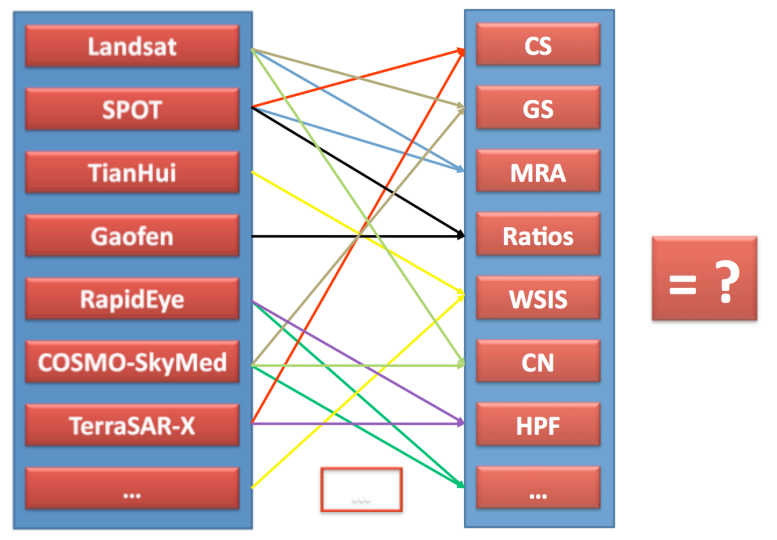

Figure 1. How to decide which fusion algorithm is yielding the best result for a certain data set?

\subsection{Concept}

The described problems led to the idea to develop a Fusion Approach Selection Tools (FAST). FAST shall provide the user with an overview of processing flows to choose from to reach the target. It will facilitate access to an optimized and improved image processing technology to support daily demands of image providers and end users. FAST shall use available images, ask for application parameters, desired information and then shall process this input in order to come out with a workflow to quickly obtain the best results. It will be designed to optimize data and image fusion techniques and provide an overview on the possible results from which the user can choose the best one by visual inspection and quantitative evaluation.

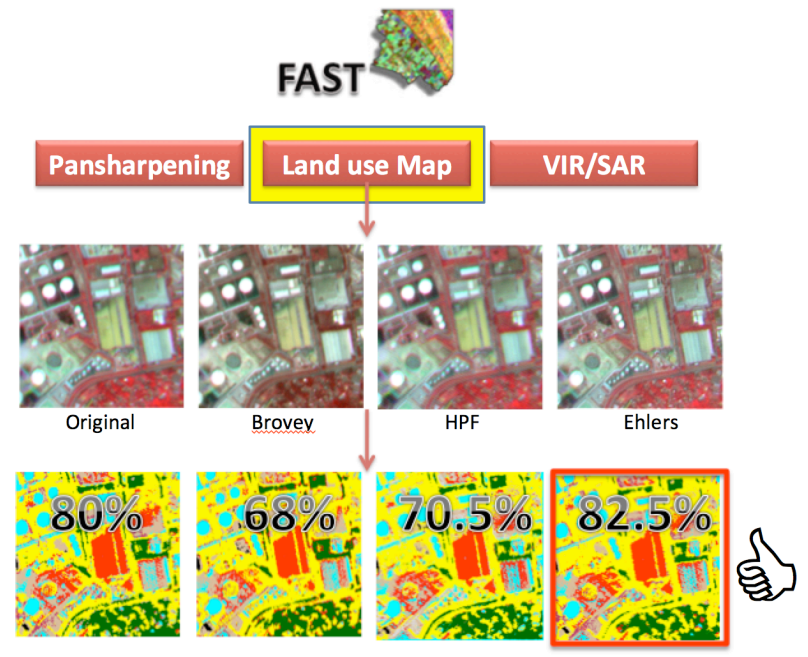

Figure 2. Concept of FAST

FAST shall enable even inexperienced users to use advanced processing methods to obtain better results without wasting time and resources. The idea behind it is explained in figure 2 . The example shows an optical data set with multispectral and panchromatic bands for land use classification. The system is supposed to display the results of in this case three different fusion techniques, i.e. BT, HPF and Ehlers, followed by a standard maximum likelihood classification. The user provides information about available

- Remote sensing data

- Fusion algorithms

- Post-processing (in this case ML classification)

- $\quad$ Anticipated result (land use map)

FAST will return quicklooks of the different fusion algorithms for visual inspection. This will be accompanied by a set of quality indices for quantitative evaluation. The user can use these criteria for the decision on the technique and process the data accordingly. The quantitative criterion provided in figure 2 is the classification accuracy achieved.

\subsection{Foundation}

The system is founded on a comprehensive database of research results serving as knowledge source. Published research results in remote sensing image fusion (Pohl and Yen 2014) and information from interviews with experts were collected, assessed and catalogued over a period of two years (Pohl 2013). Further details about this information are provided in section 3 of this article.

\subsection{Organisation}

For the development of FAST it is indispensible to obtain an overview of achievements and operational remote sensing image fusion in the different applications. Therefore the available research findings were sorted into different classes. This concerned the algorithms and their categorization (2.3.1), applications of image fusion (2.3.2), sensor types (2.3.3), evaluation criteria (2.3.4) and the development of a proper workflow (2.3.5). Currently the results of the database and questionnaire analyses are being compiled and transformed into a textbook. This leads to general guidelines and practical implementation strategies that will form the basis of FAST. The 
core system will concentrate on well-understood fusion algorithms and conventional image combinations. Later on it can be extended to more recent developments and more complex data sets.

2.3.1 Structuring fusion techniques: There have been many efforts to provide a complete overview on existing remote sensing image fusion techniques (Pohl and Van Genderen 2014; Khaleghi et al. 2013; Jinghui et al. 2010; Pohl and Van Genderen 1998). It remains an important task due to on-going development of new sensors and new algorithms. Another issue in this context is the categorization of the different techniques to allow a structured approach in choosing an appropriate algorithm (Pohl and van Genderen 2015). The system will follow the well-established five groups mentioned above. It allows accommodating all commercially available and commonly used fusion techniques without overlap. This approach fosters the understanding of image fusion technology in remote sensing for newcomers. According to the collected information from indexed journal publications the most popular algorithms are CS, MRA and lately hybrid algorithms along with arithmetic methods as shown in figure 3 . CS is very popular mostly because of its simplicity and straightforwardness. MRA has caught up popularity ranking because of the availability and accessibility of more powerful computing facilities. It has shown to produce good quality.

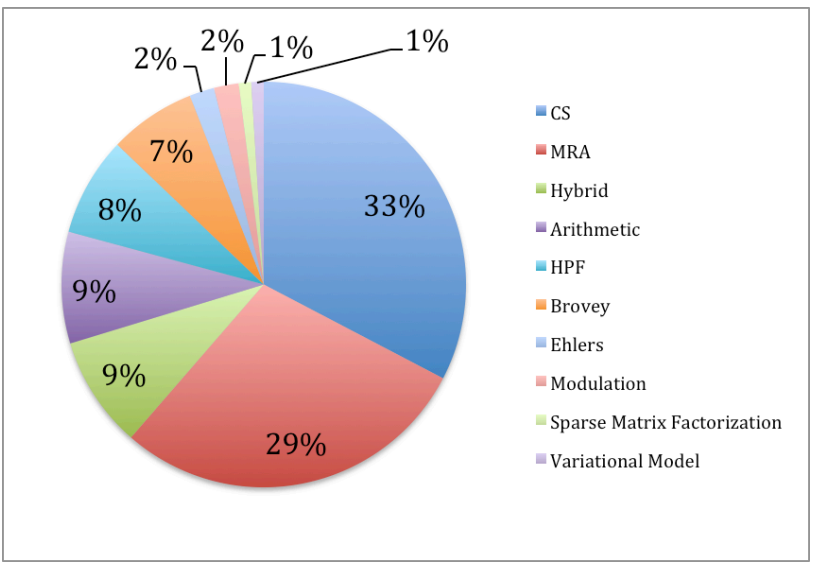

Figure 3. Popularity of algorithms from journal publications

2.3.2 Applications: Depending on the application previous research has identified optimized processing chains. Different applications call for different fusion approaches, mostly depending on the input data and the post-processing applied to extract the desired information. Successful image fusion applications are urbanization, change detection, geology, forestry, vegetation, agriculture, hazards, land cover and mapping in general. Hot topics are the monitoring of urbanization (Werner et al. 2014; Ghanbari and Sahebi 2014; Gamba 2014; Palsson et al. 2012) due to the increased spatial resolution of new sensors and precision agriculture (Wang et al. 2014; Gevaert 2014; Amorós-López et al. 2013) using hyperspectral images and radar data.

Figure 4 illustrates a possible result from FAST to support effective processing for ornamental tree classification. The system is supposed to provide a quicklook of each available and promising fusion approach for visual inspection. In combination with statistical quality parameters the user can make the choice and process the entire data set without wasting time on unsuccessful fusion examples. Figure 4 displays a comparison of hue - saturation - value (HSV), Gram-Schmidt (GS), colour normalization $(\mathrm{CN})$ and PCA fusion results of WorldView-2 multispectral and panchromatic data.

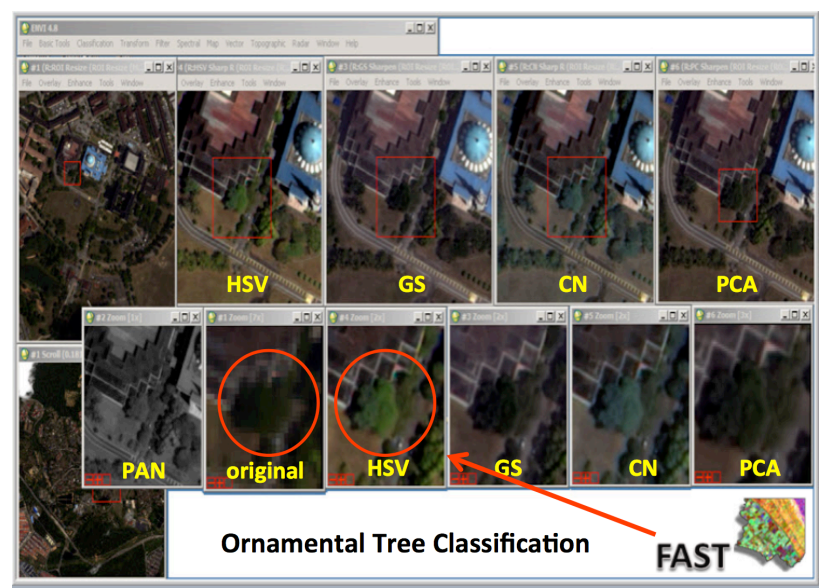

Figure 4. FAST application example

In terms of research image fusion started to become popular with geological applications in the 90's using optical and radar data. With pansharpening and the higher spatial resolution urban monitoring, land use mapping and change detection came into focus as can be seen in figures $5 \mathrm{a}$ and $5 \mathrm{~b}$. The first chart (5a) displays the percentage of occurrence of remote sensing applications in indexed journal papers. The second chart (5b) displays the use of image fusion by international experts who responded to the questionnaire.

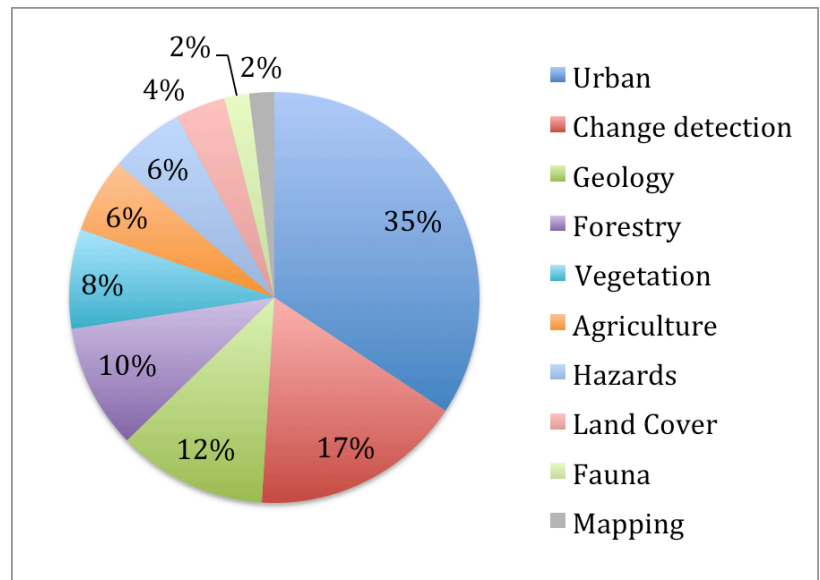

Figure 5a. Applications as published in indexed journal papers

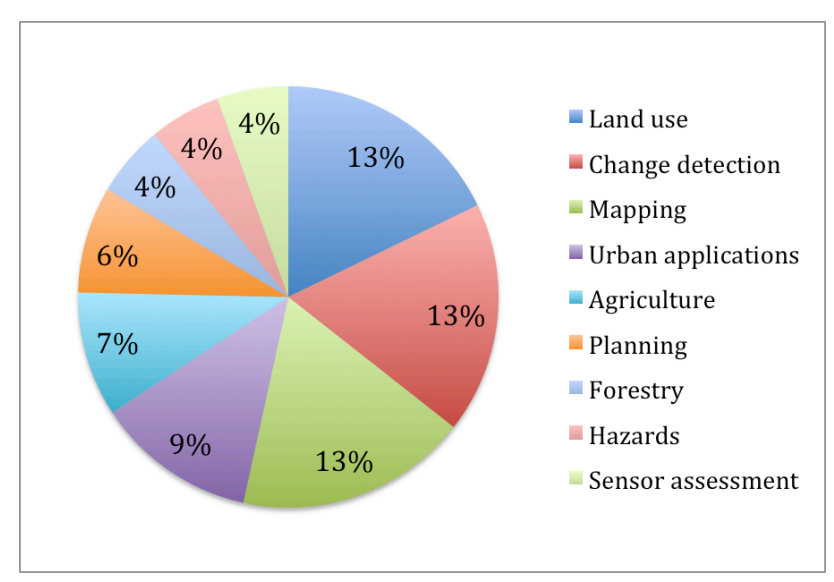

Figure 5b. Applications based on the questionnaire 
Both source groups mention change detection as a high application priority, which is obviously a strong field for remote sensing image fusion. The reason for this focus is probably that a single sensor, especially optical remote sensing data is often not available on the time needed for the survey. It is expected that natural hazards and disasters will receive more attention in the near future because international organisations have set up Internet portals to provide multi-sensor remote sensing images for humanitarian aid. More and more images become publically available, which will foster remote sensing image fusion in this important application field.

2.3.3 Sensor data: Year by year the remote sensing community takes advantage of more and more operational and sophisticated new sensors. According to the collected database IKONOS, Quickbird, Landsat and SPOT are the most popular platforms (Pohl and Yen 2014). This relates to the fact that all of them enable single-platform pansharpening. However this may change now as new research is being conducted on the integration of hyperspectral images and LiDAR (Light Detection and Ranging) data with conventional remote sensing images.

2.3.4 Evaluation criteria: There is no fusion research without quality assessment. Even though visual inspection (qualitative evaluation) is very popular it does not provide objective criteria for quality assessment. It is very much dependent on the experience of the operator. Therefore users rely on quantitative measures to analyse the quality of the fusion results. Here spectral and spatial qualities play a role, for which separate and combined indices exist. Recently researchers focused on the potential of a technique to extract the necessary information (Xin et al. 2014). Another research team identified that different aspects and different data require careful selection of the quality measure (Makarau et al. 2012).

2.3.5 Workflows: The workflow to fuse remote sensing images is mainly depending on the data and the fusion technique. The sensor defines the pre-processing steps; the following fusion delimits the necessary image processing. The workflow can be delineated precisely per fusion algorithm. If the user desires to apply a classification for mapping purposes FAST will assist and go a step further by providing unsupervised classification results quicklooks per selected fusion algorithm as previously illustrated in figure 2 .

\section{IMPLEMENTATION}

For the implementation of FAST it was necessary to assess the current state of the art in remote sensing image fusion. Therefore existing indexed journal literature of the past 15 years has been collected and classified. In the process of the research an extensive database of the publications on remote sensing image fusion has been compiled (Pohl and Yen 2014). About 850 papers describing data combinations, fusion techniques, applications and quality assessment have been catalogued to date. They were sorted into a set of categories to analyse commonalities and contradictions. Ca. 300 publications are highly relevant for remote sensing applications. The list of categories embraces applications, sensors, fusion techniques, areas of achievement, resampling, quality assessment, and open questions. In parallel a questionnaire was sent out to experts in the field (Pohl 2013). It embraced two parts: Part A requested information about general experiences obtained using remote sensing image fusion. There are seven questions collecting the expertise:
- Years of experience

- Types of images used in image fusion

- Application

- Fusion technique(s) used

- Quality assessment performed

- Image processing software

- Challenges faced

The participants were also asked which major trends they foresee, which problems still need to be resolved, and which key application areas they identified in image fusion.

Part B collected material about a particular case study to illustrate the implementation of image fusion. This information is relevant for building application cases and deriving trends. This is completed by the applications collected from the published research case studies. While collecting information on remote sensing image fusion applications it became obvious that far more research is dedicated to algorithm development than implementation for certain applications. The ratio is 220/54 of the number of algorithm/application papers. That makes more than four times more publications on fusion techniques development than applying it to real world problems.

The analysis of the collected material leads to the necessary background information to formulate the rules for FAST and design the system to be built. The general background for the system forms a spatial decision support system (SDSS). A SDSS is defined as a computer system that provides decisionmakers with an opportunity to assess and solve problems with spatial information. In the case of FAST this would refer to providing existing experience in fusing remote sensing images in a certain giving context. The system will contain information about

- Sensors,

- Fusion techniques, and

- Applications,

which are connected by established rules derived from the database and the questionnaire. These rules form general guidelines based on the experience gained in the last 20 years of remote sensing image fusion and are represented as arrows in figure 6 .

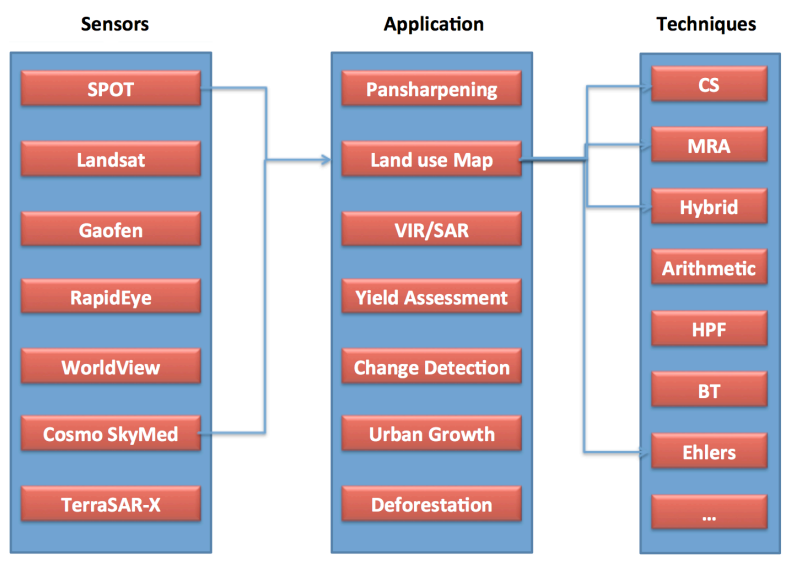

Figure 6. The arrows follow the rules stored in the SDSS.

FAST would then request the selection of a relevant subset in one of the images for the production of the quicklook. The user can then choose representative areas in the data to foresee the effect of the different fusion techniques. FAST fuses only the 
subset, outputs the quicklooks for visual inspection accompanied by established remote sensing image fusion quality indices in comparison for the user to take the final decision. An option is to finally process the data according to the choices made by the operator. This issue is secondary as long as the optimum approach to fuse the images is provided by FAST.

\section{CONCLUSION}

This paper introduces a concept for the development of a fusion approach selection tool (FAST) to assist remote sensing users in obtaining the best possible multi-sensor images. The concept is founded on research about generalization of trends in image fusion derived from the past 20 years. Two input sources were evaluated, interviews with experts and published literature from indexed international journals. Currently the acquired information is converted into guidelines and rules that serve as input for a spatial decision support system as background for FAST. The next steps involve the definition of the system and the implementation by a team of programmers. Experts interested to join the effort to develop such a system are welcome to fill in the questionnaire (Pohl 2013) and join the remote sensing image fusion discussion group on LinkedIn (Pohl 2014).

\section{ACKNOWLEDGEMENTS}

This work was supported by the Universiti Teknologi Malaysia under the grant Q.J130000.2709.00K 71.

\section{REFERENCES}

Amorós-López, J., L. Gómez-Chova, L. Alonso, et al., 2013. Multitemporal fusion of Landsat/TM and ENVISAT/MERIS for crop monitoring. International Journal of Applied Earth Observation and Geoinformation 23(1), pp. 132-141.

Baronti, S., B. Aiazzi, M. Selva, A. Garzelli, and L. Alparone, 2011. A Theoretical Analysis of the Effects of Aliasing and Misregistration on Pansharpened Imagery. Selected Topics in Signal Processing, IEEE Journal of 5(3), pp. 446-453.

Battiston, R., M. Migliaccio, and S. Stramondo, 2014. Foreword to the Special Issue on COSMO-Skymed First and Second Generation Exploitation. Selected Topics in Applied Earth Observations and Remote Sensing, IEEE Journal of 7(7), pp. 2707-2710.

Ehlers, M., 2004. Spectral characteristics preserving image fusion based on Fourier domain filtering. Paper read at Remote Sensing for Environmental Monitoring, GIS Applications, and Geology IV, at Bellingham, WA, USA.

Gamba, P., 2014. Image and data fusion in remote sensing of urban areas: Status issues and research trends. International Journal of Image and Data Fusion 5(1), pp. 2-12.

Gevaert, C., 2014. Combing hyperspectral UAV and multispectral Formosat-2 imagery for precision agriculture applications, Department of Geography and Ecosystem Science, Lund University.

Ghanbari, Z., and M. Sahebi, 2014. Improved IHS Algorithm for Fusing High Resolution Satellite Images of Urban Areas. Journal of the Indian Society of Remote Sensing 42(4), pp. 689699.
Hong, G., Y. Zhang, and B. Mercer, 2009. A wavelet and IHS integraton method to fuse high resolution SAR with moderate resolution multispectral images. Photogrammetric Engineering \& Remote Sensing 75(10), pp. 1213-1223.

Jawak, S. D., and A. J. Luis, 2013. A comprehensive evaluation of pan-sharpening algorithms coupled with resampling methods for image synthesis of very high resolution remotely sensed satellite data. Advances in Remote Sensing 2, pp. 332-344.

Jinghui, Y., J. Zhang, L. Haitao, S. Yushan, and P. Pengxiam, 2010. Pixel level fusion methods for remote sensing images: A current review. In ISPRS TC VII Symposium - 100 Years ISPRS, edited by W. S. Wagner, B. (eds.). Vienna, Austria: ISPRS.

Khaleghi, B., A. Khamis, F. O. Karray, and S. N. Razavi, 2013. Multisensor data fusion: A review of the state-of-the-art. Information Fusion 14(1), pp. 28-44.

Li, H., B. S. Manjunath, and S. K. Mitra, 1993. Multisensor image fusion using the wavelet transform. Computer Vision, Graphics, and Image Processing: Graphical Models and Image Processing 57, pp. 235-245.

Makarau, A., G. Palubinskas, and P. Reinartz, 2012. Analysis and selection of pan-sharpening assessment measures. Journal of Applied Remote Sensing 6(1), pp. 063548-1-063548-20.

Otukei, J. R., T. Blaschke, and M. Collins, 2015. Fusion of TerraSAR-x and Landsat ETM+ data for protected area mapping in Uganda. International Journal of Applied Earth Observation and Geoinformation 38, pp. 99-104.

Palsson, F., J. R. Sveinsson, J. A. Benediktsson, and H. Aanaes, 2012. Classification of Pansharpened Urban Satellite Images. Selected Topics in Applied Earth Observations and Remote Sensing, IEEE Journal of 5(1), pp. 281-297.

Pohl, C., Compilation of a remote sensing image fusion atlas 2013 [cited 07 June 2015]. Available from https://docs.google.com/forms/d/1yLfVU1EQdxoH1G9ut_OtiHf6Op6JTcV6XapCzSbg-A/viewform.

Pohl, C., 2015. Remote Sensing Image Fusion 2014 [cited 10 June 2015]. Available from http://lnkd.in/eQxNwY.

Pohl, C., and J. Van Genderen, 1998. Review article multisensor image fusion in remote sensing: concepts, methods and applications. International Journal of Remote Sensing 19(5), pp. 823-854.

Pohl, C., and J. Van Genderen, 2014. Remote sensing image fusion: an update in the context of Digital Earth. International Journal of Digital Earth 7(2), pp. 158-172.

Pohl, C., and J. Van Genderen, 2015. Structuring contemporary remote sensing image fusion. International Journal of Image and Data Fusion 6(1), pp. 3-21.

Pohl, C., and T. L. Yen, 2014. Compilation of a remote sensing image fusion atlas. In 35th Asian Conference of Remote Sensing $(A C R S)$. Nay Pyi Taw, Myanmar.

Wang, L., Y. Tian, X. Yao, Y. Zhu, and W. Cao, 2014. Predicting grain yield and protein content in wheat by fusing multi-sensor and multi-temporal remote-sensing images. Field Crops Research 164(1), pp. 178-188. 
Werner, A., C. D. Storie, and J. Storie, 2014. Evaluating SAROptical Image Fusions for Urban LULC Classification in Vancouver Canada. Canadian Journal of Remote Sensing 40(4), pp. 278-290.

Xin, H., W. Dawei, X. Junfeng, and Z. Liangpei, 2014. Quality Assessment of Panchromatic and Multispectral Image Fusion for the ZY-3 Satellite: From an Information Extraction Perspective. Geoscience and Remote Sensing Letters, IEEE 11(4), pp. 753-757.

Xu, Q., Y. Zhang, B. Li, and L. Ding, 2015. Pansharpening using regression of classified MS and pan images to reduce color distortion. IEEE Geoscience and Remote Sensing Letters 12(1), pp. 28-32.

Zhang, Y., and G. Hong, 2005. An IHS and wavelet integrated approach to improve pan-sharpening visual quality of natural colour IKONOS and QuickBird images. Information Fusion 6(3), pp. 225-234. 